\title{
Perfil Epidemiológico da Espondilodiscite Tratada Cirurgicamente
}

\section{Surgical Treated Spondylodiscitis Epidemiological Study}

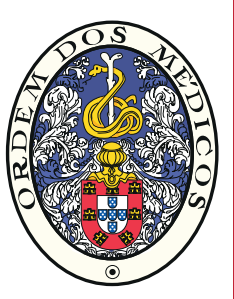

\author{
Joaquim SOARES DO BRITO ${ }^{1}$, António TIRADO1 ${ }^{1}$, Pedro FERNANDES ${ }^{1}$
}

Acta Med Port 2016 May;29(5):319-325 - http://dx.doi.org/10.20344/amp.6549

RESUMO

Introdução: O termo espondilodiscite descreve uma qualquer infeção da coluna vertebral, sendo o tratamento gold standard baseado na terapêutica médica e a indicação cirúrgica ponderada em casos particulares. Foi objetivo deste trabalho estudar o perfil epidemiológico de um grupo de doentes com espondilodiscite submetidos a tratamento cirúrgico numa mesma instituição nacional entre 1997 e 2013.

Material e Métodos: Análise epidemiológica de 85 doentes com diagnóstico de espondilodiscite submetidos a tratamento cirúrgico. Foram analisados os processos clínicos, estudos de imagem e registos informáticos.

Resultados: Foram tratados 51 doentes do género masculino e 34 do género feminino. A idade média foi de 48 anos (min: 6 - máx: 80). O segmento lombar foi o mais afetado e o Mycobacterium tuberculosis o agente etiológico mais frequente. A distribuição do número de casos ao longo dos anos manteve-se aproximadamente constante, com discreto aumento da incidência do Staphylococus aureus e diminuição do número de casos sem agente identificado. Trinta e nove doentes apresentavam abcesso paravertebral e 17 lesão neurológica concomitante, a maioria no segmento torácico e em relação com infeção tuberculosa. Dez doentes apresentavam imunodepressão importante.

Discussão: Nesta série verificou-se um predomínio da infeção tuberculosa, no género masculino e em indivíduos jovens. Enquanto o número de casos/ano das discites tuberculosas se mantém constante, as infeções a Staphylococus aureus parecem ganhar preponderância. O abcesso paravertebral e a lesão neurológica constituíram uma complicação frequente da infeção tuberculosa.

Conclusão: As infeções da coluna vertebral com necessidade cirúrgica persistem como patologia relevante. Mycobacterium tuberculosis e Staphylococcus aureus representam os principais agentes etiológicos, parecendo existir uma incidência crescente deste último agente.

Palavras-chave: Discite/cirurgia; Discite/epidemiologia; Procedimentos Ortópedicos.

\section{ABSTRACT}

Introduction: The term spondylodiscitis aims to describe any spinal infection. Medical treatment is the gold standard; nevertheless, surgical treatment can be indicated. The aim of this work was to study the epidemiological profile in a group of patients with spondylodiscitis surgically treated in the same medical institution between 1997 and 2013.

Material and Methods: Eighty five patients with spondylodiscitis were surgically treated in this period. The authors analysed clinical data and image studies for each patient.

Results: We treated 51 male and 34 female patients with an average age of 48 years old ( $\min : 6$ - max: 80). The lumbar spine was more often affected and Mycobacterium tuberculosis the most frequent pathogen. The number of cases through the years has been grossly stable, with a slight increase of dyscitis due to Staphylococcus aureus and decrease of the dyscitis without pathogen identification Paravertebral abscess was identified in 39 patients and 17 had also neurological impairment, mostly located in the thoracic spine and with tuberculous aetheology. Immunosuppression was documented in 10 patients.

Discussion: In this epidemiologic study we found a tuberculous infection, male gender and young age predominance. Despite a relative constant number of patients operated over the years, pyogenic infections due to Staphylococcus aureus seems to be uprising. Paravertebral abscess and neurological impairment are important dyscitis complications, especially in tuberculous cases.

Conclusion: Spinal infections requiring surgical treatment are still an important clinical condition. Mycobacterium tuberculosis and Staphylococcus aureus represent the main pathogens with a growing incidence for the latest.

Keywords: Discitis/surgery; Discitis/epidemiology; Orthopedic Procedures.

\section{INTRODUÇÃO}

O termo espondilodiscite corresponde a uma infeção que envolve o disco intervertebral, o corpo vertebral ou os elementos posteriores da vértebra. A etiologia desta condição clinica pode ser piogénica (bacteriana), granulomatosa (tuberculosa, brucelosa ou fúngica) ou parasitária. ${ }^{1}$ Apesar de ser considerada uma doença rara, a espondilodiscite tem apresentado incidência crescente. ${ }^{2}$ Este incremento na incidência está relacionado com diversos fatores, entre os quais podemos enumerar o número significativo de doentes utilizadores de drogas endovenosas; o maior número de doentes em hemodiálise por longos períodos; a crescente esperança média de vida em doentes com múltiplas comorbilidades e imunodeprimidos; e um crescimento exponencial da cirurgia de coluna vertebral. ${ }^{1,3} \mathrm{~A}$ par destes factores existe inegavelmente uma maior acuidade diagnóstica, que em conjunto gera um número crescente de casos registados. ${ }^{1,3,4}$

As três vias preferenciais para o desenvolvimento da infeção são a via hematogenea, a inoculação direta e a propagação a partir de infeções dos tecidos adjacentes. ${ }^{1}$ O Staphylococus aureus continua a ser descrito como o agente etiológico mais frequente da espondilodiscite

1. Serviço de Ortopedia e Traumatologia. Hospital de Santa Maria. Centro Hospitalar Lisboa Norte. Lisboa. Portugal.

$\triangle$ Autor correspondente: Joaquim Soares do Brito. joaquimsoaresdobrito@gmail.com

Recebido: 24 de abril de 2015 - Aceite: 19 de setembro de 2015 | Copyright @ Ordem dos Médicos 2016 
piogénica, no entanto, o agente mais frequentemente implicado à escala global é o Micobacterium tuberculosis. ${ }^{1,3,5-7}$ Outros agentes etiológicos como a Pseudomonas aeruginosa, Staphylococus coagulase-negativos e Streptococcus, são mais frequentes em doentes utilizadores de drogas endovenosas. ${ }^{1,3}$ Infeções fúngicas ou por parasitas são extremamente raras. ${ }^{8}$

Estima-se atualmente que a incidência anual de espondilodiscite na Europa poderá oscilar entre 0,4 e 2,8 novos casos por 100.000 habitantes ao ano, no entanto, estes dados estão sujeitos à variabilidade que deriva dos critérios de inclusão utilizados na sua estimativa. ${ }^{3} \mathrm{Na}$ literatura mais recente, é relatada a maior incidência de casos de espondilodiscite entre os indivíduos do género masculino, principalmente entre os mais idosos, devido, provavelmente, à elevada taxa de comorbilidades existente entre os homens europeus acima dos 60 anos. . $^{3,9,10}$

Clinicamente, a espondilodiscite pode manifestar-se por dor, febre, deformidade cifótica, abcesso paravertebral ou inclusivamente lesão neurológica de instalação progressiva. Na fase inicial da doença o diagnóstico pode ser difícil, pelo que é fundamental a avaliação clínica, laboratorial e imagiológica. ${ }^{1}$

O tratamento médico baseado em quimioterapia anti-infeciosa mantem-se como o gold standard no tratamento de qualquer caso de espondilodiscite, colocando-se indicação cirúrgica perante a evidência de instabilidade vertebral, falência da terapêutica médica, presença de deformidade importante e progressiva, ou em alternativa, perante a existência de abcesso paravertebral/epidural com extensão considerável ou lesão neurológica de novo. ${ }^{1}$

Dada a escassez de dados nacionais relativamente ao universo da espondilodiscite, os autores deste trabalho procuraram definir o perfil epidemiológico de um grupo de doentes com diagnóstico de espondilodiscite (piogénica,

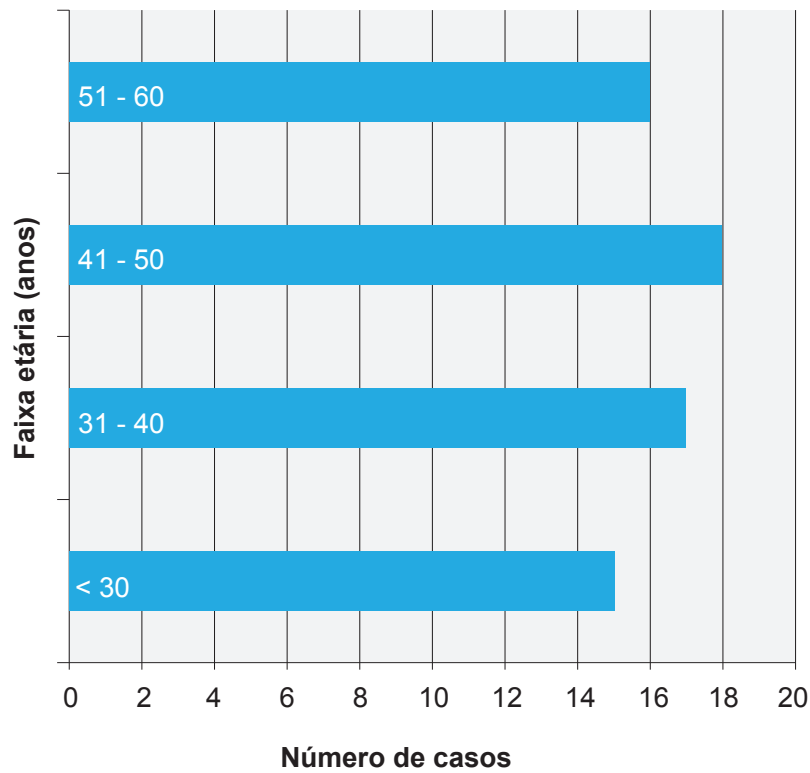

Figura 1 - Distribuição do número de casos de espondilodiscite por faixa etária granulomatosa ou de outra etiologia) submetidos a tratamento cirúrgico numa mesma instituição nacional num período de 17 anos, decorrente entre 1997 e 2013.

\section{MATERIAL E MÉTODOS}

Foi realizada uma análise epidemiológica dos doentes com diagnóstico de espondilodiscite, independentemente da sua etiologia, submetidos a tratamento cirúrgico no nosso Serviço de Ortopedia e Traumatologia entre o ano de 1997 e 2013 (17 anos). Foram identificados 85 casos de espondilodiscite operados, coadjuvando a quimioterapia anti-infeciosa (antibacteriana ou anti-tuberculosa) implementada em todos os doentes, sob orientação do departamento de doenças infeciosas.

Foram analisados os processos clínicos, estudos imagiológicos arquivados e registos constantes no processo informático de cada doente. Para a avaliação de cada parâmetro em estudo foram excluídos os doentes cuja informação não foi possível obter a partir das fontes consultadas. Foram recolhidos dados demográficos, dados relativos à caracterização da lesão, avaliada a presença de lesão neurológica, isolamento de agente etiológico e comorbilidades associadas.

Como critérios para implementação do tratamento cirúrgico, os autores consideraram: a falência do tratamento médico com quimioterapia anti-infeciosa; a presença de lesão neurológica; a presença de abcesso paravertebral de grandes dimensões com ou sem extensão para o espaço epidural; existência de instabilidade vertebral.

\section{RESULTADOS}

A idade média dos doentes foi de 48 anos (mín: 6 máx: 80 ), sem diferenças significativas entre o número de espondilodiscites registadas nos vários grupos etários (Fig. 1). Dos 85 doentes identificados, 51 eram do género

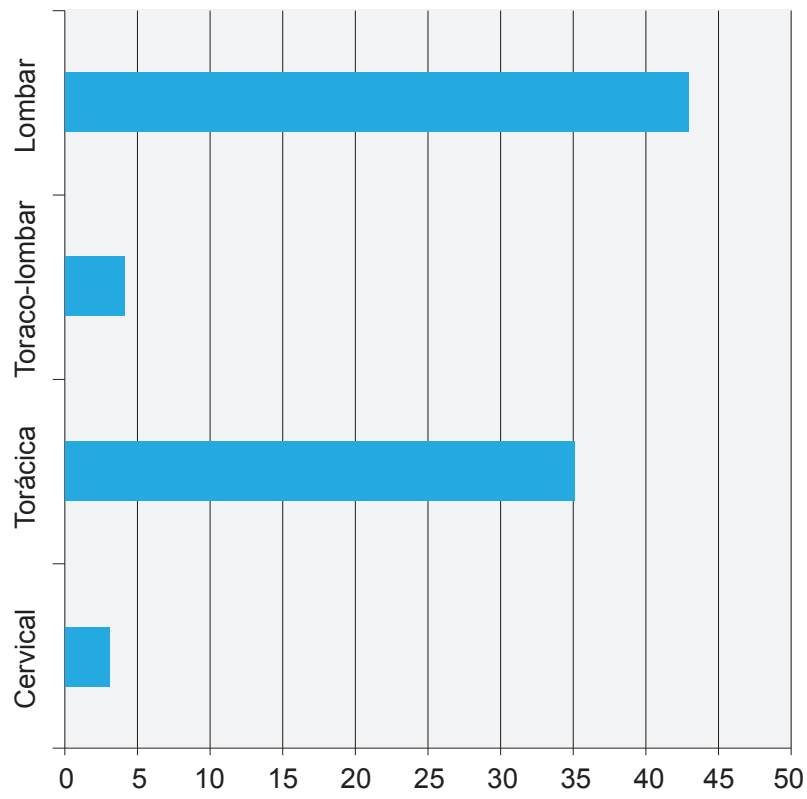

Figura 2 - Distribuição da espondilodiscite por segmento anatómico afetado 
masculino e 34 do género feminino; 64 doentes leucodérmicos e 21 melanodérmicos. O segmento lombar foi o mais frequentemente afetado com 43 casos, seguido da coluna torácica com 35 casos; na transição toraco-lombar foram identificados quatro casos e a coluna cervical somente esteve implicada em três ocasiões (Fig. 2).

Relativamente ao agente etiológico, foi possível isolar Mycobacterium tuberculosis em 43 casos; Staphylococcus aureus em 15; Staphylococcus aureus meticilino-resistente em três; Streptococcus pyogenes numa ocasião e ainda dois casos de infeção a Brucella spp. Não foi possível identificar agente etiológico em 21 ocasiões (Fig. 3). A etiologia tuberculosa esteve presente em todos os casos com idade pediátrica.

Quando se relacionou a etiologia da espondilodiscite com o segmento da coluna vertebral afetado não se verificou diferença significativa para a infeção a Mycobacterium tuberculosis (dos 43 casos, 20 encontravam-se localizados a nível torácico e 19 a nível lombar); ou para a infeção a Staphylococcus aureus (dos 15 casos oito foram identificados na coluna torácica e seis na coluna lombar). 0 maior número de casos sem agente etiológico identificado surgiu a nível lombar (quatro casos não identificados na coluna torácica comparativamente a 15 casos não identificados no segmento lombar).

A avaliação da distribuição do número de casos ao longo dos anos não demonstrou evidência de um número crescente de espondilodiscites com indicação para tratamento cirúrgico (Fig. 4). De igual modo, verifica-se um número relativamente constante de espondilodiscites a Mycobacterium tuberculosis (Fig. 4), enquanto se tem assistindo a uma tendência crescente para espondilodiscites

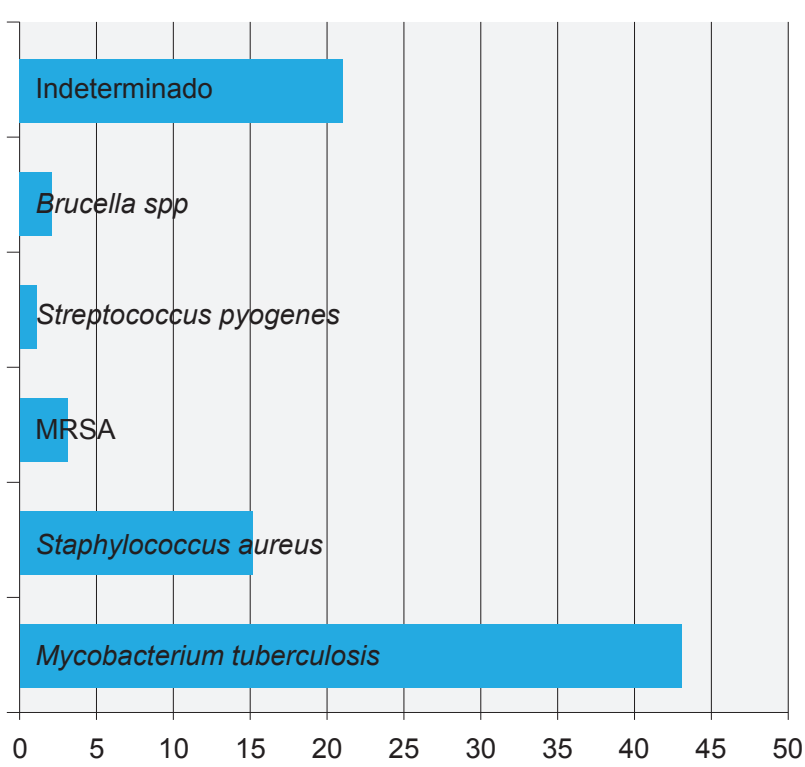

Figura 3 - Número de espondilodiscites por agente etiológico

de etiologia piogénica, nomeadamente a Staphylococcus aureus, verificando-se simultaneamente menor número de casos de discites sem agente etiológico identificado (Fig. 4).

À data do diagnóstico, 39 doentes apresentavam abcesso paravertebral, tendo sido identificados 17 doentes com lesão neurológica. A avaliação da etiologia do abcesso paravertebral revelou que dos 39 casos identificados, 25 eram por infeção tuberculosa, sete por infeção piogénica e sete de etiologia indeterminada (Fig. 5).

A maioria dos casos com envolvimento neurológico surgiram na coluna torácica (11 casos), seguida da coluna

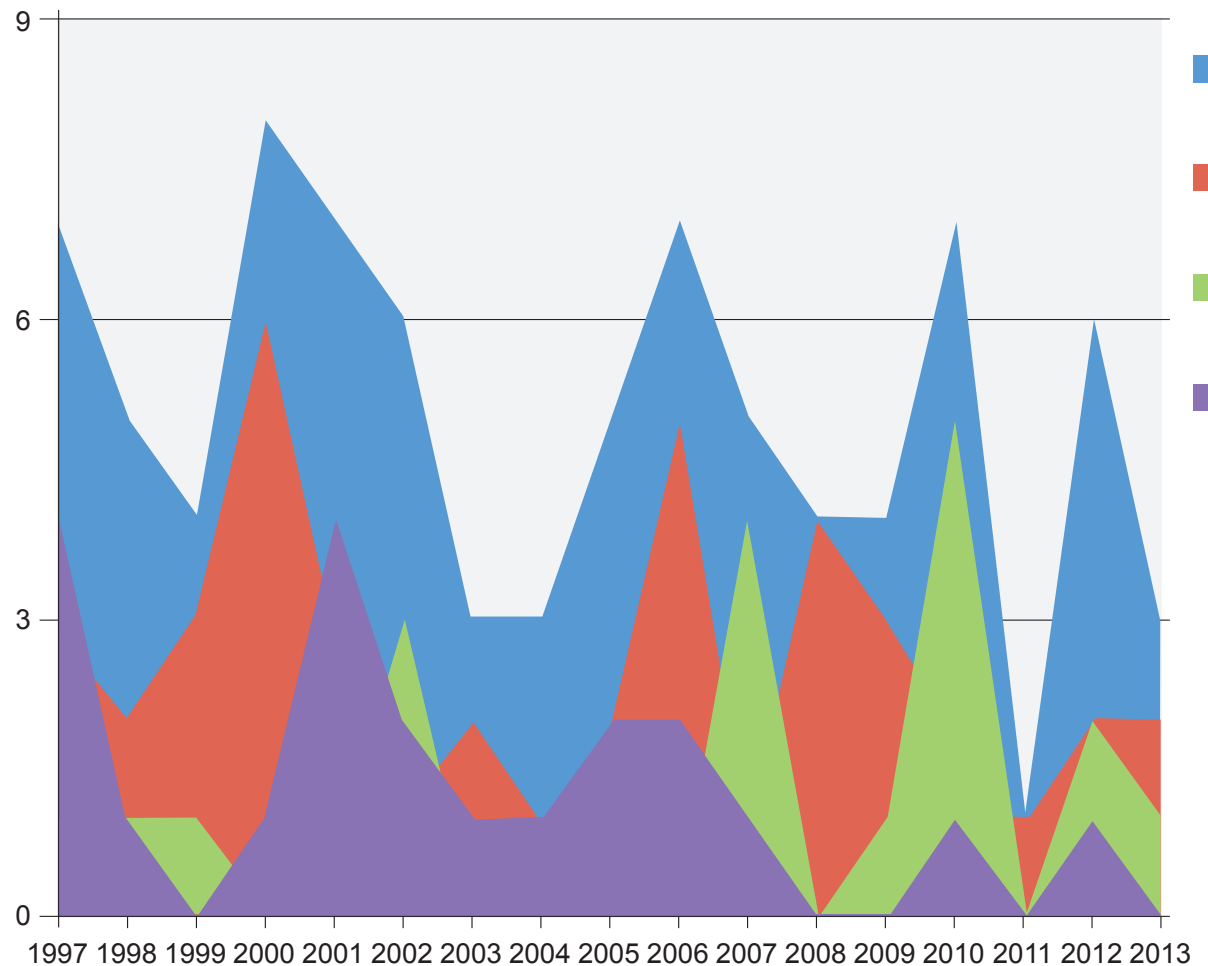

Total

Mycobacterium tuberculosis

Staphylococcus aureus / MRSA

Indeterminada

Figura 4 - Número de casos/ano por agente etiológico isolado 


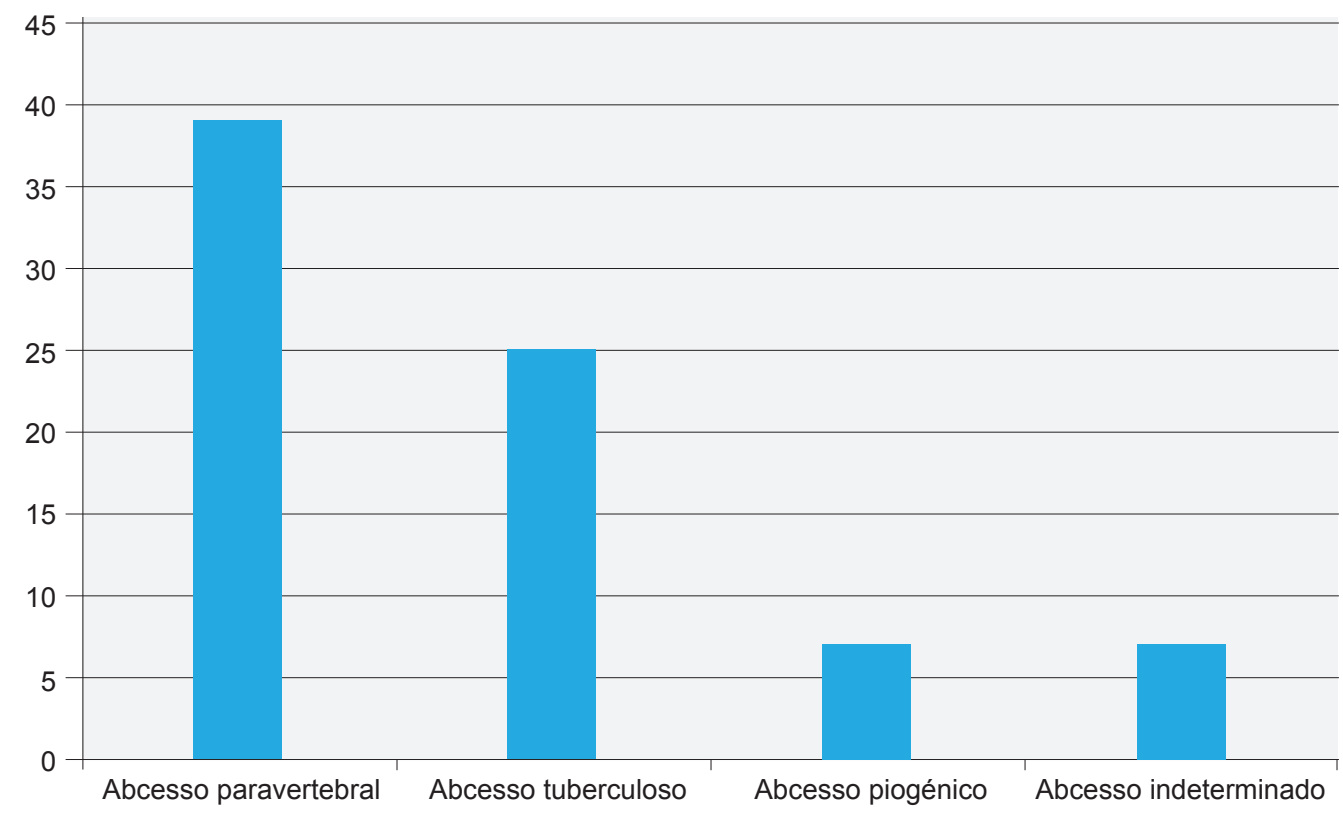

Figura 5 - Presença de abcesso paravertebral de acordo com a etiologia infeciosa

lombar (quatro casos) e um caso ao nível da transição toraco-lombar. A presença de lesão neurológica surgiu maioritariamente na infeção turberculosa (12 casos) seguida de infeção piogénica (cinco casos).

Dos 85 doentes tratados, quatro eram seropositivos para o $\mathrm{VIH}$, sendo identificados outros seis doentes com comorbilidades importantes a condicionar imunodepressão, nomeadamente doença oncológica, alcoolismo crónico, toxicodependência endovenosa e diabetes mellitus tipo 2 (Tabela 1).

\section{DISCUSSÃO}

A espondilodiscite corresponde a uma condição clinica pouco frequente, embora com incidência crescente nos países ocidentais. ${ }^{1,11,12}$ As razões para o crescimento da sua incidência são várias, destacando-se a crescente esperança média de vida em doentes com múltiplas comorbilidades; maior número de doentes imunodeprimidos; e um crescimento exponencial da cirurgia de coluna vertebral. , $^{1,3,11,12}$ Estas infeções são mais frequentes entre os indivíduos do género masculino e recentemente têm sido descritas incidências e prevalências crescentes entre uma faixa etária mais idosa. ${ }^{11,12} \mathrm{~A}$ nossa série corrobora a maior predominância deste diagnóstico entre o género masculino, contudo, o mesmo não se aplica à distribuição por faixa etária da população afetada. Explicamos os nossos resultados pelo facto da nossa população incluir um importante número de doentes jovens provenientes dos países africanos de língua oficial portuguesa, contrariando a tendência verificada nos restantes países ocidentais onde a população afetada é sobretudo a mais idosa.

A opção terapêutica habitual perante o diagnóstico de espondilodiscite recai na terapêutica anti-infeciosa, constituindo o tratamento gold standard desta condição clinica.
O grupo de doentes aqui apresentado representa um grupo particular, que para além do tratamento médico foi submetido a tratamento cirúrgico. A indicação cirúrgica aplica-se perante situações específicas e tem sido nossa conduta propor cirurgia em casos de falência do tratamento médi$\mathrm{co}$, na presença de lesão neurológica de novo, se existir abcesso paravertebral de grandes dimensões, com ou sem extensão para o espaço epidural, e perante a existência de critérios de instabilidade vertebral. ${ }^{1,6,7}$ Os doentes representados nesta série obedeceram a estes critérios cirúrgicos e mantiveram quimioterapia anti-infeciosa concomitante.

Neste grupo de doentes verificou-se que os casos de espondilodiscite tratada cirurgicamente surgem predominantemente na coluna lombar (43 dos 85 casos), o que vai de encontro ao já amplamente descrito na literatura. ${ }^{1} \mathrm{De}$ modo global, esta série não permite confirmar o incremento da incidência que tem sido descrito, verificando-se oscilações pouco significativas do número global de espondilodiscites ao longo dos anos. Lembramos, no entanto, que esta série engloba apenas doentes tratados cirurgicamente, não incluindo os doentes tratados medicamente.

O Staphylococcus aureus continua a ser a etiologia piogénica mais frequente e o agente mais importante nos países desenvolvidos. No entanto, a avaliação dos números globais, continua a colocar a etiologia tuberculosa como principal causa desta condição clinica. ${ }^{1} \mathrm{Na}$ série de doentes aqui apresentada verifica-se um predomínio da infeção tuberculosa, achado que entra em conflito com as etiologias habitualmente relatadas como mais frequentes noutros países ocidentais. ${ }^{9,11-14}$ Uma vez mais, este resultado pode refletir o fato da nossa instituição constituir centro de referenciação para os países africanos de língua oficial portuguesa e onde a tuberculose é endémica. No entanto, quando analisamos o número de casos/ano por etiologia 
podemos verificar que o número de espondilodiscites tuberculosas se tem mantido constante, enquanto as infeções piógénicas, particularmente por Staphylococus aureus, (incluindo os meticilino-resistentes), parecem ganhar preponderância. Outras etiologias como as infeções estreptocócicas ou a Brucella spp são, sem dúvida mais raras, e este fato foi elucidado pelos poucos casos registados nes- ta série. É de realçar o número relevante de casos onde não foi possível identificar o agente etiológico apesar das várias culturas realizadas. Todavia, registamos um número decrescente de espondilodiscites sem agente identificado nos últimos anos, resultado de uma atitude, desde sempre, muito proativa na obtenção do diagnóstico etiológico.

A avaliação deste grupo de doentes permitiu confirmar

Tabela 1 - Caraterização da população em estudo

\begin{tabular}{|c|c|c|c|c|c|c|c|c|}
\hline Doente & Idade & Género & Coluna & Etiologia & Abcesso & ASIA & Comorbilidades & Abordagem cirúrgica \\
\hline Doente 1 & 26 & $\mathrm{M}$ & $\mathrm{L}$ & Indeterminada & Não & $E$ & & Posterior \\
\hline Doente 2 & 48 & $\mathrm{~F}$ & $\mathrm{~L}$ & Indeterminada & Sim & $E$ & & Posterior \\
\hline Doente 3 & 38 & M & $\mathrm{L}$ & Indeterminada & Não & $E$ & & Posterior \\
\hline Doente 4 & 67 & $\mathrm{~F}$ & $\mathrm{~L}$ & M. tuberculosis & $\operatorname{Sim}$ & $E$ & & Anterior \\
\hline Doente 5 & 48 & M & $\mathrm{L}$ & M. tuberculosis & Não & $E$ & & Dupla \\
\hline Doente 6 & 52 & M & $\mathrm{T}$ & M. tuberculosis & Não & $E$ & & Posterior \\
\hline Doente 7 & 70 & $\mathrm{~F}$ & $\mathrm{~L}$ & Indeterminada & Sim & $E$ & & Anterior \\
\hline Doente 8 & 38 & $\mathrm{M}$ & $\mathrm{L}$ & S. Aureus & Não & $E$ & $\mathrm{VIH}+$ & Anterior \\
\hline Doente 9 & 38 & $\mathrm{M}$ & $\mathrm{L}$ & Indeterminada & Não & $E$ & & Anterior \\
\hline Doente 10 & 43 & M & $\mathrm{L}$ & M. tuberculosis & $\operatorname{Sim}$ & $E$ & $\mathrm{VIH}+$ & Anterior \\
\hline Doente 11 & 64 & $\mathrm{M}$ & $\mathrm{L}$ & M. tuberculosis & $\operatorname{Sim}$ & $E$ & & Anterior \\
\hline Doente 12 & 63 & $\mathrm{~F}$ & $\mathrm{~L}$ & Brucella spp & Não & $E$ & & Anterior \\
\hline Doente 13 & 59 & M & $\mathrm{L}$ & M. tuberculosis & Não & $E$ & & Anterior \\
\hline Doente 14 & 24 & $\mathrm{M}$ & $\mathrm{T}$ & M. tuberculosis & Sim & $E$ & & Anterior \\
\hline Doente 15 & 16 & $\mathrm{M}$ & $\mathrm{T}$ & M. tuberculosis & Sim & $E$ & & Posterior \\
\hline Doente 16 & 34 & $M$ & $\mathrm{~T}$ & S. Aureus & Não & $E$ & & Posterior \\
\hline Doente 17 & 40 & M & $\mathrm{T}$ & M. tuberculosis & Sim & $E$ & $\mathrm{VIH}+$ & Dupla \\
\hline Doente 18 & 58 & $\mathrm{~F}$ & $\mathrm{~T}$ & Indeterminada & Sim & $E$ & & Dupla \\
\hline Doente 19 & 45 & $\mathrm{~F}$ & $\mathrm{~L}$ & M. tuberculosis & Não & $E$ & & Dupla \\
\hline Doente 20 & 58 & M & $\mathrm{T}$ & M.tuberculosis & $\operatorname{Sim}$ & $E$ & & Anterior \\
\hline Doente 21 & 66 & $\mathrm{~F}$ & $\mathrm{~L}$ & M. tuberculosis & Não & $E$ & & Anterior \\
\hline Doente 22 & 54 & M & $\mathrm{T}$ & M. tuberculosis & Não & $\mathrm{E}$ & & Dupla \\
\hline Doente 23 & 36 & $\mathrm{M}$ & $\mathrm{L}$ & M.tuberculosis & Sim & $E$ & & Anterior \\
\hline Doente 24 & 32 & $\mathrm{~F}$ & $\mathrm{~L}$ & M. tuberculosis & $\operatorname{Sim}$ & $E$ & & Dupla \\
\hline Doente 25 & 21 & $\mathrm{~F}$ & $\mathrm{~T}$ & Indeterminada & Não & $E$ & & Posterior \\
\hline Doente 26 & 44 & M & C & Indeterminada & Não & $E$ & & Posterior \\
\hline Doente 27 & 34 & M & $\mathrm{T}$ & M. tuberculosis & $\operatorname{Sim}$ & $E$ & & Dupla \\
\hline Doente 28 & 53 & $\mathrm{~F}$ & $\mathrm{~L}$ & Indeterminada & Não & $\mathrm{E}$ & & Posterior \\
\hline Doente 29 & 32 & $\mathrm{M}$ & $\mathrm{T}$ & Indeterminada & Sim & $E$ & & Dupla \\
\hline Doente 30 & 70 & $\mathrm{M}$ & $\mathrm{L}$ & M. tuberculosis & Sim & $\mathrm{E}$ & & Dupla \\
\hline Doente 31 & 37 & $\mathrm{M}$ & $\mathrm{T}$ & M. tuberculosis & Não & $E$ & & Posterior \\
\hline Doente 32 & 41 & M & $\mathrm{T}$ & M. tuberculosis & Não & $E$ & & Dupla \\
\hline Doente 33 & 47 & $\mathrm{~F}$ & $\mathrm{~L}$ & Indeterminada & Não & $E$ & & Posterior \\
\hline Doente 34 & 21 & $\mathrm{M}$ & $\mathrm{L}$ & Indeterminada & $\operatorname{Sim}$ & $E$ & & Dupla \\
\hline Doente 35 & 57 & $M$ & $\mathrm{~L}$ & S. Aureus & Não & $E$ & & Dupla \\
\hline Doente 36 & 65 & $\mathrm{M}$ & $\mathrm{L}$ & S. Aureus & Não & $E$ & & Dupla \\
\hline Doente 37 & 47 & $\mathrm{~F}$ & $\mathrm{C}$ & S. Aureus & Não & $E$ & & Dupla \\
\hline Doente 38 & 66 & $M$ & $\mathrm{~T}-\mathrm{L}$ & Indeterminada & Sim & $E$ & & Anterior \\
\hline Doente 39 & 39 & $\mathrm{M}$ & $\mathrm{T}$ & M. tuberculosis & Sim & B & $\mathrm{VIH}+$ & Dupla \\
\hline Doente 40 & 25 & $\mathrm{M}$ & $\mathrm{L}$ & M. tuberculosis & Sim & $E$ & & Dupla \\
\hline Doente 41 & 48 & M & $\mathrm{T}-\mathrm{L}$ & M. tuberculosis & Sim & $E$ & & Dupla \\
\hline Doente 42 & 55 & $\mathrm{~F}$ & $\mathrm{~L}$ & Brucella spp & Sim & $E$ & & Dupla \\
\hline Doente 43 & 56 & M & $\mathrm{T}$ & Indeterminada & Não & $E$ & & Dupla \\
\hline
\end{tabular}


Tabela 1 - Caraterização da população em estudo (continuação)

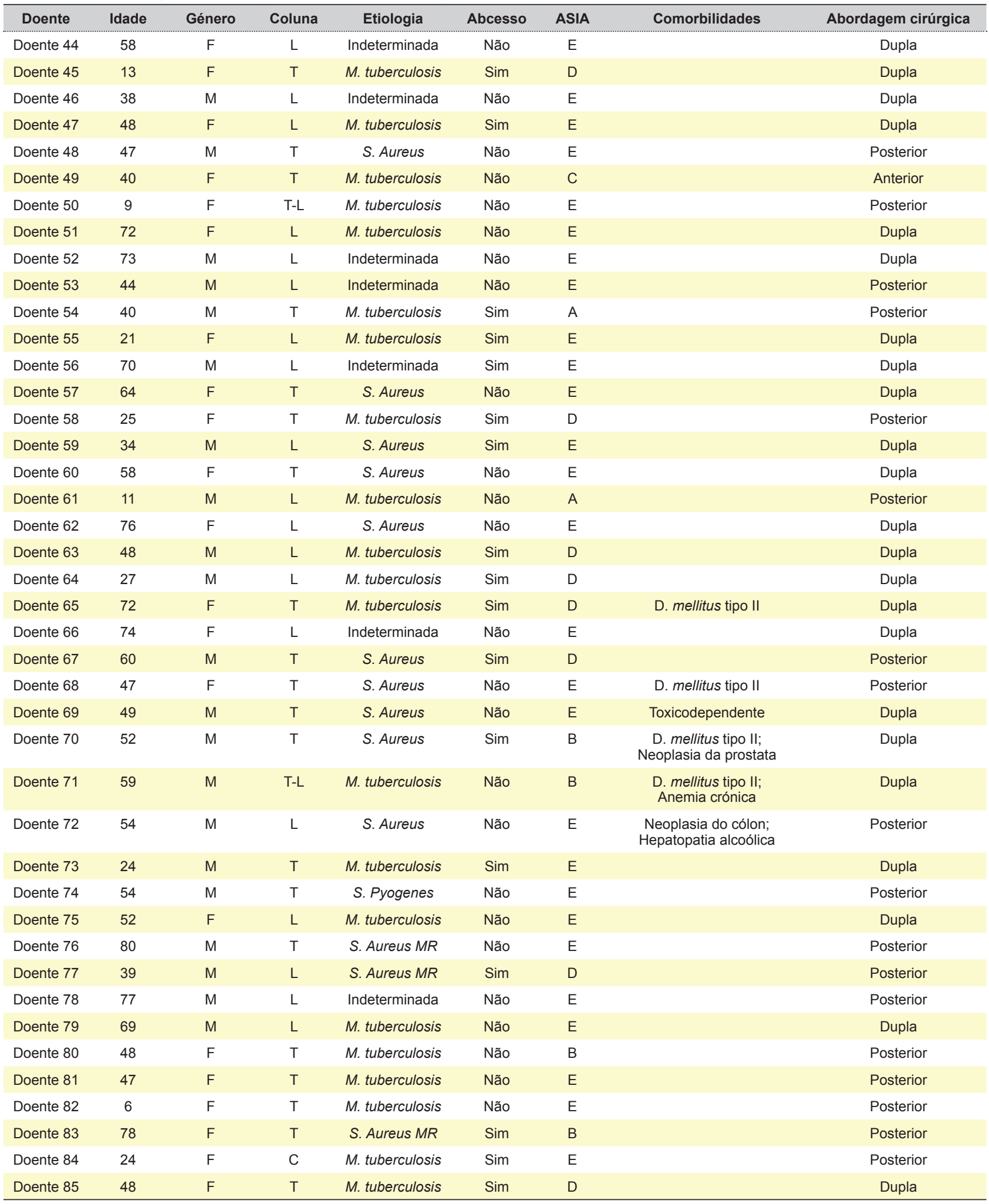

M: masculino; F: feminino; C: cervical; T: torácico; TL: toraco-lombar; L: lombar;

o abcesso paravertebral como complicação frequente da espondilodiscite, particularmente os casos associados à etiologia tuberculosa ( 25 dos 40 casos identificados). A infeção piogénica produziu abcesso paravertebral num menor número de casos (quatro casos das 15 espondilo- discites a Staphylococus aureus) e habitualmente com menor volume.

A presença de lesão neurológica constituiu igualmente um achado significativo na presença de espondilodiscite (17 casos) e está particularmente relacionado com a 
infeção tuberculosa (12 casos entre os 17 doentes com lesão neurológica). Segundo Hodgson et al, a etiologia da paraplegia na tuberculose com menos de dois anos de evolução estava relacionada com a doença ativa, onde a compressão é causada por pús ou tecido de granulação, enquanto a paraplegia com mais de dois anos de evolução está habitualmente relacionada com a existência de compressão óssea gerada pela deformidade acentuada. ${ }^{14,15}$

Dos 85 doentes tratados cirurgicamente com diagnóstico de espondilodiscite, apenas se identificaram quatro casos de seropositividade para o vírus da imunodeficiência humana e outros seis casos de doentes com comorbilidades importantes a condicionar imunossupressão subjacente, nomeadamente, doença oncológica ativa, alcoolismo crónico, toxicodependência endovenosa e diabetes mellitus tipo 2. Destes números se infere uma taxa global de doentes imunossuprimidos discretamente superior a $10 \%$, no entanto, seis destes dez casos correspondem a doentes tratados nos últimos cinco anos. Este achado traduz-se numa taxa de $25 \%$ de doentes com imunossupressão, se tivermos em conta apenas os casos mais recentes. Assim, torna-se evidente que este grupo particular de doentes está a aumentar entre aqueles que mais contribuem para a incidência crescente da espondilodiscite. ${ }^{11-14}$

Em nosso entender, este trabalho constitui uma contribuição válida para o melhor conhecimento da epidemiologia das infeções da coluna vertebral em Portugal, nomeadamente daquelas que requerem intervenção cirúrgica para a sua resolução. No entanto, parece-nos fundamental continuar a monitorizar esta entidade clinica, de modo a compreendermos ainda melhor a sua realidade no contexto nacional.

\section{REFERÊNCIAS}

1. Guerado E, Cerván AM. Surgical treatment of spondylodiscitis. An update. Int Orthop. 2012;36:413-20.

2. Jensen AG, Espersen F, Skinhøj P, Rosdahl VT, Frimodt-Møller N. Increasing frequency of vertebral osteomyelitis following Staphylococcus aureus bacteraemia in Denmark 1980-1990. J Infect. 1997;34:113-8.

3. Fantoni M, Trecarichi EM, Rossi B, Mazzotta V, Di Giacomo G, Nasto LA, et al. Epidemiological and clinical features of pyogenic spondylodiscitis. Eur Rev Med Pharmacol Sci. 2012;16:S2-7.

4. Carragee EJ. Pyogenic Vertebral Osteomyelitis. J Bone Joint Surg Am. 1997;79:874-80.

5. Colmenero JD, Jiménez-Mejías ME, Reguera JM, Palomino-Nicas $\mathrm{J}$, Ruiz-Mesa JD, Márquez-Rivas J, et al. Tuberculous vertebral osteomyelitis in the new millennium: still a diagnostic and therapeutic challenge. Eur J Clin Microbiol Infect Dis. 2004;23:477-83.

6. Luk KDK. Tuberculosis of the spine in the new millennium. Euro Spine J. 1999;8:338-45.

7. Mclain RF, Isada C. Spinal tuberculosis deserves a place on the radar screen. Cleve Clin J Med. 2004;71:537-9.

8. Frazier DD, Campbell DR, Garvey TA, Wiesel S. Fungal infections of the spine. J Bone Joint Surg Am. 2001;83:560-60.

9. Grammatico L, Baron S, Rusch E, Lepage B, Surer N, Desenclos JC, et al. Epidemiology of vertebral osteomyelitis (VO) in France: analysis of

\section{CONCLUSÃO}

As infeções da coluna vertebral com necessidade de tratamento cirúrgico persistem como patologia relevante no contexto atual. Neste grupo particular são afetados fundamentalmente doentes do género masculino, existindo uma elevada presença de abcesso paravertebral e lesão neurológica, acarretando morbilidade importante. O Mycobacterium tuberculosis e o Staphylococcus aureus permanecem como os principais agentes infeciosos, parecendo existir uma incidência crescente deste última etiologia.

\section{PROTECÇÃO DE PESSOAS E ANIMAIS}

Os autores declaram que os procedimentos seguidos estavam de acordo com os regulamentos estabelecidos pelos responsáveis da Comissão de Investigação Clínica e Ética e de acordo com a Declaração de Helsínquia da Associação Médica Mundial.

\section{CONFIDENCIALIDADE DOS DADOS}

Os autores declaram ter seguido os protocolos do seu centro de trabalho acerca da publicação de dados.

\section{CONFLITOS DE INTERESSE}

Os autores declaram não terem qualquer conflito de interesse relativamente ao presente artigo.

\section{FONTES DE FINANCIAMENTO}

Os autores declaram não ter recebido subsídios ou bolsas para a elaboração do artigo.

hospital-discharge data 2002-2003. Epidemiol Infect. 2008;136:653-60. 10. Skaf GS, Domloj NT, Fehlings MG, Bouclaous $\mathrm{CH}$, Sabbagh AS, Kanafani ZA, et al. Pyogenic spondylodiscitis: an overview. J Infect Public Health. 2010;3:5-16.

11. Citak M, Backhaus M, Kälicke T, Hilal Z, Muhr G, Frangen TM, et al. Myths and facts of spondylodiscitis: An analysis of 183 cases. Acta Orthop Belg. 2011;77:535.

12. Beronius, M, Bergman B, Andersson R. Vertebral Osteomyelitis in Goteborg, Sweden: A Retrospective Study of Patients During 1990-95. Scand J Infect Dis. 2001;33:527-32.

13. D'agostino C, Scorzolini L, Massetti AP, Carnevalini M, D'Ettorre G, Venditti $\mathrm{M}$, et al. A seven-year prospective study on spondylodiscitis: epidemiological and microbiological features. Infection. 2010;38:102-7.

14. Soares do Brito J, Batista N, Tirado A, Fernandes P. Surgical treatment of spinal tuberculosis: an orthopedic service experience. Acta Med Port. 2013;26:349-56.

15. Medical Research Council (MRC) Working Party on Tuberculosis of Spine. A 10-year assessment of a controlled trials comparing debridement and anterior spinal fusion in the management of tuberculosis of the spine on standard chemotherapy in Hong Kong. J Bone Joint Surg Br. 1982;64:393-8. 